\title{
Prognostic study of risk stratification among Japanese patients with ischemic heart disease using gated myocardial perfusion SPECT: J-ACCESS study
}

Tsunehiko Nishimura $\cdot$ Kenichi Nakajima •

Hideo Kusuoka • Akira Yamashina •

Shigeyuki Nishimura

Published online: 26 July 2008

(C) Springer-Verlag 2008

Erratum to: Eur J Nucl Med Mol Imaging

DOI 10.1007/s00259-007-0608-x

Eur J Nucl Med (2008) 35:319-328

In Table 1, the entry for "Family history of coronary artery disease" should read "394/3,325 (11.9\%)" instead of "1,871/3,968 (47.2\%)".

The same data were mistakenly copied from the line above.

The online version of the original can be found at http://dx.doi.org/ 10.1007/s00259-007-0608-x.

T. Nishimura $(\bowtie)$

Department of Radiology, Graduate School of Medical Science,

Kyoto Prefectural University of Medicine,

465 Kajiicho, Kawara-machi, Hirokoji,

Kamigyo-ku 602-8566 Kyoto, Japan

e-mail: nisimura@koto.kpu-m.ac.jp

H. Kusuoka

Osaka National Hospital,

Osaka, Japan

A. Yamashina

Second Department of Internal Medicine,

Tokyo Medical University Hospital,

Tokyo, Japan

S. Nishimura

Division of Cardiology, Saitama Medical School Hospital,

Saitama, Japan

K. Nakajima

Department of Biotracer Medicine, Kanazawa University

Graduate School of Medical Science,

Kanazawa, Japan

e-mail: nakajima@med.kanazawa-u.ac.jp 